\title{
Fibrosis in dysthyroid eye disease
}

\author{
Marian Ludgate ${ }^{1}$
}

Received: 21 October 2019 / Accepted: 28 October 2019 / Published online: 16 December 2019

(c) The Royal College of Ophthalmologists 2019

\begin{abstract}
Dysthyroid eye disease is a rare condition, mainly found in people with Graves' hyperthyroidism. Autoimmune responses to thyroid/orbit shared antigens drive extensive tissue remodelling. This includes excess adipogenesis and over-production of extra-cellular matrix, which both tend to occur in the earlier 'active' inflammatory stages of disease. With time these give way to fibrosis, which has a profound impact on eye motility and may be life-long. Progress has been made in identifying the shared autoantigen(s) and the role of specific $\mathrm{T}$ cells and autoantibodies in remodelling, which have facilitated development of novel therapies. However relatively little is known of the autoimmune processes under-pinning fibrosis and currently there are no adequate medical treatments.
\end{abstract}

\section{Dysthyroid eye disease-vital statistics}

Dysthyroid eye disease is also known as thyroid eye disease (TED) or Graves' orbitopathy (GO) and has a prevalence of $\sim 10 / 10,000$ in Europe, which is reducing. It is an autoimmune disease and consequently more common in women, although when present in men, can be more severe [1].

The main signs and symptoms include periorbital oedema, lid lag, proptosis, chemosis, reduced ocular motility, reduced visual acuity, diplopia and optic neuropathy [2]. The severity and activity of TED can be assessed using a variety of scoring systems whose relative merits have been recently reviewed [3]. The Clinical Activity Score (CAS) and VISA scale (Vision, Inflammation, Strabismus, and Appearance) reflect disease activity, whereas the EUGOGO, NOSPECS (no symptoms/signs, only signs, soft tissue involvement, proptosis, extraocular muscle involvement, corneal involvement, and sight loss), VISA-VSA scales and Ophthalmopathy Index measure severity.

The natural course of TED, as described by Rundle's curve, is unique to each patient; all experience an initial inflammatory phase, of varying duration and severity,

Marian Ludgate

Ludgate@ cardiff.ac.uk

1 Institute of Infection \& Immunity, School of Medicine, Cardiff University, Cardiff, UK which can be alleviated by glucocorticoids. The disease eventually burns out and becomes amenable to corrective surgery, although decompression may be necessary in the active phase to avoid optic neuropathy and blindness [4]. TED has a significant negative impact on quality of life, which has been evaluated using the specific GO-QOL questionnaire [5]. Smoking is a major risk factor both for the incidence and severity of the condition; improved clinical outcomes are associated with patients who are able to quit [6].

\section{Evidence for an autoimmune aetiology?}

The infiltration of immune cells into an organ or tissue is indicative of autoimmunity. Such studies in TED have been hampered by the paucity of suitable material and the fact that surgical specimens tend to be available only at the later 'inactive' stages of disease. However, an overview of available studies indicates that both CD4+ and CD8 $+\mathrm{T}$ cells as well as B cells are present in the majority of orbits examined. T cells tend to be CD45R+, suggesting recent activation. Bahn et al. described predominantly Th- 1 cells in early TED, evolving to Th- 2 as disease progressed [7]. A more recent report demonstrated that the level of infiltration correlated with disease activity [8]. Newly differentiated CD14+ macrophages are found in early disease [9], whilst monocytes and mast cells have also been identified and found to stimulate proliferation of orbital fibroblasts (OF). 


\section{Which types of dysthyroidism-how can thyroid disease affect the eye?}

More than $90 \%$ of TED patients have concurrent Graves' disease (GD). In GD, autoantibodies to the thyrotropin receptor (TSHR) mimic the biological action of thyroid stimulating hormone (TSH) resulting in hyperthyroidism [10]. Up to $50 \%$ of GD patients also have TED, which is severe in 3-5\%. However, some people with TED have hypothyroidism whilst a small proportion are euthyroid [11]. Of interest, we have demonstrated the presence of TSHR autoantibodies even in euthyroid TED, although they lack biological activity when measured using conventional assays [12].

To be considered a relevant thyroid/orbit shared autoantigen it should be (a) expressed in the orbit; (b) disease activity should correlate with antibody or $\mathrm{T}$ cell-mediated immune response to the autoantigen; (c) TED-like disease should be induced using the autoantigen; (d) antagonism of the autoantigen should improve disease.

Many investigators have demonstrated that the TSHR is expressed in orbital tissues. Our own studies reported that the TSHR is expressed in orbital fat [13] and is upregulated during the lineage specific differentiation of mesenchymal stem cells into adipocytes [14], discussed below. TED incidence and severity correlates with titres of autoantibodies to the TSHR [15]. Furthermore, it is possible to induce signs and symptoms of TED by immunising mice with the human TSHR-A subunit and the model has been reproduced in more than one laboratory $[16,17]$.

The fact that some TED patients are euthyroid and/or apparently free of TSHR autoantibodies has led some investigators to seek additional thyroid/orbit shared autoantigens. The IGF1R and its signalling pathways have been the subject of intense investigation, often with conflicting results. Smith et al. have demonstrated that autoantibodies to the IGF1R in TED patients produce a range of effects, including enhanced OF proliferation, increased secretion of inflammatory cytokines and elevated production of GAGs [18]. Others find little evidence for IGF1R autoantibodies being elevated in TED [19, 20], whilst Neumann and coworkers suggest that cross-talk between the TSHR and IGF1R, following activation of the TSHR, has a central role in TED pathogenesis [21]. This model could be compatible with the dramatic improvements in proptosis following treatment with an IGF1R antagonist, teprotumumab, reported by Smith et al. [22].

Neither the TSHR nor IGF1R fulfil all criteria; disease activity correlating with immune reactivity to the IGF1R is lacking as is evidence that TSHR antagonism improves TED. However, treatment of an unusual thyroid cancer patient who also had GD and TED, with a human monoclonal thyroid blocking antibody (TBAB) improved these disorders. The results are also compatible with my view that we do not need any additional autoantigens-but maybe we have yet to identify TED-specific TSHR autoantibodies [12].

\section{Pathogenetic mechanisms operating in TED}

The expansion of tissues within the confines of the bony orbit is thought to account for many of the signs and symptoms of disease. In the early active phase this is predominantly via proliferation of $\mathrm{OF}$; their over-production of extra-cellular matrix (ECM) and excess adipogenesis [23]. Several authors have reported that the basal rate of $\mathrm{OF}$ proliferation is higher in patients with TED compared with normal orbits. Proliferation can be stimulated by secreted factors (e.g., IGF1, PDGF, and IL-4) or direct cell-cell contact (e.g., CD40-CD154) with immune cells infiltrating the inflamed orbit. Cytokines secreted by macrophages and mast cells, such as TGF- $\beta$, stimulate production of ECM components including hyaluronan (HA) and other glycosaminoglycans. Adipogenesis is triggered in vitro by hormone cocktails including agents to increase cAMP, insulin, dexamethasone and PPAR- $\gamma$ agonists such as rosiglitazone. High affinity endogenous PPAR $-\gamma$ agonists have yet to be identified, potential ligands such as lysophosphatidic acid (16:0 and 18:1) and 15-deoxy-PGJ2 have low affinity [23].

The possibility that activation of the TSHR and/or IGF1R is able to modulate adipogenesis and production of ECM components has also been investigated (see below).

\section{What is different about orbital fibroblasts? What is the cellular target of the autoimmune response?}

OFs are derived from neural crest, unlike fibroblasts in other depots which mainly have a mesenchymal origin. Recent studies investigated the stem-cell properties of $\mathrm{OF}$ and demonstrated that the majority are $\mathrm{CD} 90+$ and $\mathrm{CD} 45-$, which are positive and negative markers of stem cells, respectively. Furthermore, OF were able to undergo adipogenesis, chondrogenesis and myogenesis in vitro in appropriate differentiation cocktails $[24,25]$. An additional feature of OF is their expression of CD40, making them susceptible to activation by $\mathrm{CD} 40 \mathrm{~L}$ which is present on $\mathrm{T}$ cells. The CD40-CD40L/CD154 bridge results in HA generation by $\mathrm{OF}$ and cytokine release by both OF and T cells [26].

In most people with TED OF are exposed to hyperthyroidism, i.e., elevated circulating thyroxine and triiodothyronine. However thyroid hormones were shown to inhibit TGF- $\beta$ signalling and hence reduce the fibrotic processes in liver and skin, both in vitro and in animal models. The TGF- $\beta$ promoter contains thyroid hormone response elements [27]. 
TED patients' OF are also exposed to TSHR and IGF1R agonists, TSAB and increased IGF1, respectively, which activate the relevant signal cascades. Our own studies indicated that TSHR activation stimulated the early phases of adipogenesis [28] and the group of Bahn reported that a human monoclonal TSAB was able to replace insulin, when inducing fat-cell differentiation in vitro [29]. We also compared gene-expression profiles of fibroblasts experiencing TSHR activation with controls lacking this stimulus. The top two upregulated genes in the activated cell population were HAS-1 and HAS-2, HA synthase 1 and 2, respectively, which encode the enzymes leading to production of HA [30].

Activation of the IGF1R leads to increased OF proliferation, secretion of pro-inflammatory cytokines and production of ECM but, as discussed above, whether IGF1R autoantibodies exist which are able to stimulate signalling via the receptor remains an open question [18].

\section{CD90/Thy-1; more than a surface marker?}

As noted above, the majority of OF are $\mathrm{CD} 90+$; $\mathrm{CD} 90$ is also known as Thy-1, which is a glycoprotein attached to the cell surface via a GPI anchor, hence it is connected to the cell via lipid rafts. Thy-1 is heavily glycosylated and has a molecular weight of $25-37 \mathrm{kD}$; as an 'outward-facing molecule' it is able to integrate a variety of extra-cellular signals. To facilitate this Thy-1 participates in several signalling cascades, including those involving receptor tyrosine kinases, $\mathrm{Ca}^{++}$flux and $\mathrm{G}$ protein coupled receptors (GPCR, the TSHR is an example of a GPCR). Thy-1 also interacts with the ECM, notably integrins and syndecan and loss of Thy- 1 expression is associated with re-programming [31].

This last is relevant to TED, in which Thy-1+ OF have been shown to respond to TGF $\beta$ stimulation by acquiring $\alpha$ smooth muscle actin ( $\alpha$-SMA) expression and a myofibroblast phenotype. In contrast Thy-1- OF respond to proadipogenic factors such as cAMP and PPAR $\gamma$ ligands and differentiate into adipocytes (fat cells, [32]).

Another feature of Thy-1 of particular significance to TED is its role as a mechano-transducer, which is achieved by its formation of trimolecular complexes with $\alpha$ and $\beta$ integrins [33]. Expansion of the orbital contents in TED by adipogenesis and over-production of ECM, within the confined bony orbit, leads to increased orbital pressure; could this influence fibrosis?

\section{What activates fibrosis in TED?}

In common with fibroblasts in other locations such as skin and lung, fibrosis in OF is upregulated by TGF $\beta$, a cytokine secreted by many cells including macrophages and fibroblasts themselves. Most in vitro models of TED pathogenesis have employed two-dimensional monolayer cultures but Bailly and colleagues have applied threedimensional systems comprising co-cultures of various cell types in collagen gels, whose composition can be varied. In the presence of TGF $\beta$, OF from TED patients had increased contractile properties and proliferation compared with healthy $\mathrm{OF}$ [34]. To investigate the effect of pressure, $\mathrm{OF}$ were cultured in the three-dimensional model and pressure, equivalent to $28 \mathrm{~mm}$ mercury and of the magnitude experienced in TED, was applied. The OF from both TED and controls, started to acquire lipid droplets, suggesting that spontaneous adipogenesis had been induced, simply by increasing the pressure. The Thy-1-low population preferentially accumulated lipid, whose precise composition is being ascertained. In subsequent experiments, macrophages were introduced into the three-dimensional cultures, this time at atmospheric pressure. There was no lipid accumulation but OF morphology was changed with increases in expression of F-actin and HA production, in an IGF1R independent manner. The macrophages also promoted a contractile phenotype in the OF but no increase in expression of $\alpha$-SMA. All these effects were more marked in TED than healthy control OF and mediated in part by PI3kinase [35].

In recent years it has been recognised that several $\mathrm{T}$ cell subsets are implicated in autoimmunity. In addition to wellcharacterised Th-1 (cell-mediated immunity) and Th-2 (humoral immunity) cells, increased numbers of the Th- 17 subset have been detected at the site of autoimmune lesions, e.g., multiple sclerosis plaques [36]. Fang et al. reported that circulating Th-17 cells, and one of their cytokines IL17A, were increased in TED patients compared with healthy controls. In addition, TED orbits were infiltrated by CD4+ and CD8+ cells and displayed higher expression of IL17receptor and IL17A which were associated with fibrotic change. Subsequent in vitro studies revealed that IL17A stimulated production of ECM in OF, via the MAPK pathway. IL17A also stimulated pro-inflammatory cytokine production (e.g., IL6 and IL8) by OF, which would exacerbate lymphocytic infiltration. Finally, a product isolated from traditional Chinese medicine (Vialinin A) was found to inhibit Rory T, a transcription factor which drives Th-17 differentiation, and thus reduced their numbers [37].

The authors then investigated the effects of IL17A on Thy-1+/CD90+ OF and Thy-1-/CD90- OF. Their results indicated that IL17A increased TGF $\beta$-induced fibrosis in the former but inhibited PPAR $\gamma$ ligand-induced adipogenesis in the latter. However, IL17A promoted secretion of proinflammatory cytokines by both $\mathrm{OF}$ subsets, especially prostaglandin $\mathrm{E}_{2}$, which in turn increased differentiation of Th-17 cells [38]. 


\section{T cells and the gut microbiota-what can in vivo models tell us?}

Differentiation of Th- 17 cells, which promote autoimmunity, is closely related to formation of regulatory $\mathrm{T}$ cells (Treg), which dampen autoimmune responses. Both depend on TGF $\beta$ with IL23 and TNF $\alpha$ being required for differentiation of Th-17; the balance between Th-17 and Treg can predispose an individual to autoimmune disease [39].

Much TED research in recent years has focussed on the end stages of disease and relatively little progress has been made in understanding the mechanisms which break tolerance to the TSHR. Several hypotheses propose that microorganisms might be implicated, either via molecular mimicry or bystander activation [40]. These hypotheses have been modified by the recognition that commensal organisms in the gut microbiota secrete metabolites, which influence the phenotype of $\mathrm{T}$ cells in the gut associated lymphoid tissue. Approximately $50 \%$ of circulating $\mathrm{T}$ cells reside within the gut at any time and studies in murine models of autoimmunity and human disease suggest that the gut microbiota may influence the balance between inflammatory Th-17 and anti-inflammatory Treg [41, 42].

In other autoimmune conditions, in vivo models have provided insight into the processes leading to loss of tolerance. My own experience with a TSHR-induced model (genetic immunisation) of GD/TED was frustrating, whilst we were able to transfer disease to naive female BALB/c mice using TSHR primed $\mathrm{T}$ cells in one location [43], we were not able to reproduce the model in another laboratory [44]; neither animal unit was specific pathogen free. Berchner-Pfannschmidt et al. have been able to reproduce a model [17] established by Moshkelgosha in Paul Banga's lab [16]. The model uses genetic immunisation and in vivo electroporation of the TSHR and although immunised mice developed signs of GD and a TED-like disease, there were some differences e.g., higher levels of thyroxine in one centre, increased TSHR autoantibodies in the other.

Analysis of the gut microbiota in the two centres revealed significant differences in $\alpha$ and $\beta$ diversity and we identified disease-associated taxonomies, which support a role for the gut microbiota in shaping the autoimmune response [45]. Subsequent experiments employed antibiotic (vancomycin), probiotic (LAB4, two strains of Lactobacilli and two strains of Bifidobactera) or human TED faecal material transfer (hFMT) to modify the gut microbiota in TSHR immunised mice. Vancomycin treatment significantly reduced gut microbiota richness and diversity compared with all other groups, Lab4 and hFMT had more subtle effects; hFMT induced a human TED-like gut microbiota. Incidence and severity of induced thyroid pathology (TSAB, elevated T4, hyperplastic thyroid) and orbitopathy were increased by Lab4 and hFMT treatment but significantly reduced by vancomycin. In orbital lymph nodes CD25+ Treg numbers were significantly lower/ higher in antibiotic and probiotic treated animals, respectively. The manipulation strategies successfully modified the gut microbiota in the early-stage of life, with an impact on TSHR-induced disease. The reduced thyroid and orbital pathology, combined with diminished Tregs, in vancomycin treated mice support a role for the gut microbiota in promoting TED.

\section{Do we see changes in the gut microbiota in patients?}

The experiments described above were part of a larger project to investigate the role of the gut microbiome in GD and TED http://www.indigo-iapp.eu/ and which has also analysed the gut microbiota composition of human subjects. We observed similar perturbations in people with GD and TED as mice with TSHR-induced disease; for example, reduced Firmicutes (F) phylum counts; significantly increased Bacteroidetes (B) phylum counts; significantly increased F:B ratio. Actinobacter phylum counts were significantly higher in people with mild TED than in GD patients free of eye disease. However, it may be difficult to distinguish the contributions of endocrine (hyperthyroidism) and immune (TSAB) factors to the changes observed.

The Firmicutes phylum includes Clostridium species and these butyrate producing bacteria have been implicated in a mouse model of hepatic fibrosis [46]. Furthermore, species in the nasal and sinus microbiomes may secrete metabolites which influence orbital inflammation and fibrosis.

Of note, changes in the gut microbiome due to smoking overlapped those identified for TED but not in GD patients or healthy controls. Smoking is the single biggest known environmental risk factor for TED incidence and severity [6] and is known to affect the gut microbiota composition [47].

\section{Concluding comments}

Fibrosis remains one of the biggest challenges facing patients with TED and the clinicians who manage them. It causes disturbance to eye motility which has a major negative impact on quality of life but we are not aware of any ongoing or pending trials to address this process.

The fact that TGF $\beta$ is required for the differentiation of Th-17 cells, Treg (whose balance influences autoimmunity) and myofibroblasts (which are directly responsible for fibrosis) suggests that it may be a useful target for treatment.

However, I am aware of long-standing studies by Paul Meyer investigating antibiotic treatment of TED patients found to harbour Staphylococcus aureus infection; the results are eagerly anticipated. 
Finally, resolution of fibrosis depends on interactions between CD90/Thy-1 and Fas to promote apoptosis [48], is TED a disease of apoptosis resistant myofibroblasts?

Acknowledgements I am sincerely grateful to the Cambridge Ophthalmological Society and organizers of the symposium for inviting me to participate once again in this excellent meeting; special thanks of course to Louise.

\section{Compliance with ethical standards}

Conflict of interest The author declares that she has no conflict of interest.

Publisher's note Springer Nature remains neutral with regard to jurisdictional claims in published maps and institutional affiliations.

\section{References}

1. Perros P, Hegedüs L, Bartalena L, Marcocci C, Kahaly GJ, Baldeschi L, et al. Graves' orbitopathy as a rare disease in Europe: a European Group on Graves' Orbitopathy (EUGOGO) position statement. Orphanet J Rare Dis. 2017;12:72. https://doi.org/10. 1186/s13023-017-0625-1.

2. Draman MS, Ludgate M. Thyroid eye disease an update. Expert Rev Ophthalmol. 2016. https://doi.org/10.1080/17469899.2016. 1202113.

3. Barrio-Barrio J, Sabater AL, Bonet-Farriol E, Velazquez-Villoria A, Galofre JC. Graves' Ophthalmopathy: VISA versus EUGOGO classification, assessment, and management. J Ophthalmol. 2015;2015:249125 https://doi.org/10.1155/2015/249125.

4. Wiersinga WM, Kahaly GJ. Graves' orbitopathy: a multidisciplinary approach. 3rd ed. Karger; Basel; 2017.

5. Terwee CB, Gerding MN, Dekker FW, Prummel MF, Wiersinga WM. Development of a disease specific quality of life questionnaire for patients with Graves' ophthalmopathy: the GO-QOL. Br J Ophthalmol. 1998;82:773-9.

6. Wiersinga WM. Smoking and thyroid. Clin Endocrinol. 2013;79:145-51. https://doi.org/10.1111/cen.12222.

7. Bahn RS. Graves' ophthalmopathy. N Engl J Med. 2010;362: 726-38. https://doi.org/10.1056/NEJMra0905750.

8. Rotondo Dottore G, Torregrossa L, Caturegli PP, Ionni I, Sframeli A, Sabini E, et al. Association of T and B cells infiltrating orbital tissues with clinical features of Graves' orbitopathy. JAMA Ophthalmol. 2018;136:613-9. https://doi.org/10.1001/jamaophtha lmol.2018.0806.

9. Virakul S, van Steensel L, Dalm VA, Paridaens D, van Hagen PM, Dik WA. Orbit-infiltrating mast cells, monocytes, and macrophages produce PDGF isoforms that orchestrate orbital fibroblast activation in Graves' ophthalmopathy. J Clin Endocrinol Metab. 2012;97:E400-408. https://doi.org/10.1210/jc.20112697.

10. Paschke R, Ludgate M. The thyrotropin receptor in thyroid disease. N Engl J Med. 1997;337:1675-81.

11. Marcocci C, Bartalena L, Bogazzi F, Panicucci M, Pinchera A. Studies on the occurrence of ophthalmopathy in Graves' disease. Acta Endocrinol. 1989;120:473-8.

12. Metcalfe R, Jordan N, Watson P, Gullu S, Wiltshire M, Crisp M, et al. Demonstration of $\operatorname{IgG}, \operatorname{IgA}$ and $\operatorname{IgE}$ autoantibodies to the human thyrotropin receptor using flow cytometry. J Clin Endocrinol Metab. 2002;87:1754-61.
13. Crisp M, Lane C, Halliwell M, Wynford-Thomas D, Ludgate M. Thyrotropin receptor transcripts in human adipose tissue. J Clin Endocrinol Metab. 1997;82:2003-5.

14. Starkey KJ, Heufelder A, Baker G, Joba W, Evans M, Davies S, et al. Adipose thyrotrophin receptor expression is elevated in Graves' and thyroid eye diseases ex vivo and indicates adipogenesis in progress in vivo. J Mol Endocrinol. 2003;30:369-80.

15. Eckstein A, Plicht M, Lax H, Neuhäuser M, Mann K, Lederbogen $\mathrm{S}$, et al. Thyrotropin receptor autoantibodies are independent risk factors for Graves' opthalmopathy and help to predict severity and outcome of the disease. J Clin Endocrinol Metab. 2006;91:3464-70. https://doi.org/10.1210/jc.2005-2813

16. Moshkelgosha S, So PW, Deasy N, Diaz-Cano S, Banga JP. Cutting edge: retrobulbar inflammation, adipogenesis, and acute orbital congestion in a preclinical female mouse model of Graves' orbitopathy induced by thyrotropin receptor plasmid-in vivo electroporation. Endocrinology. 2013;154:3008-15. https://doi. org/10.1210/en.2013-1576.

17. Berchner-Pfannschmidt U, Moshkelgosha S, Diaz-Cano S, Edelmann B, Görtz G-E, Horstmann M, et al. Comparative assessment of female mouse model of Graves' orbitopathy under different environments, accompanied by proinflammatory cytokine and Tcell responses to thyrotropin hormone receptor antigen. Endocrinology. 2016;157:1673-82. https://doi.org/10.1210/en.2015-1829.

18. Smith TJ, Janssen J. Insulin-like growth factor-I receptor and thyroid-associated ophthalmopathy. Endocr Rev. 2018; https:// doi.org/10.1210/er.2018-00066.

19. Minich WB, Dehina N, Welsink T, Schwiebert C, Morgenthaler NG, Köhrle J, et al. Autoantibodies to the IGF1 receptor in Graves' orbitopathy. J Clin Endocrinol Metab. 2013;98:752-60. https://doi.org/10.1210/jc.2012-1771.

20. Marino M, Rotondo Dottore G, Ionni I, Lanzolla G, Sabini E, Ricci D, et al. Serum antibodies against the insulin-like growth factor-1 receptor (IGF-1R) in Graves' disease and Graves' orbitopathy. J Endocrinol Investig. 2019;42:471-80. https://doi.org/ 10.1007/s40618-018-0943-8.

21. Krieger CC, Neumann S, Place RF, Marcus-Samuels B, Gershengorn MC. Bidirectional TSH and IGF-1 receptor cross talk mediates stimulation of hyaluronan secretion by Graves' disease immunoglobins. J Clin Endocrinol Metab. 2015;100:1071-7.

22. Smith TJ, George J, Kahaly MD, Daniel G, Ezra MD, James C, et al. Teprotumumab for thyroid-associated ophthalmopathy. N Engl J Med. 2007;376:1748-61. https://doi.org/10.1056/NEJMoa 1614949.

23. Taylor P, Zhang L, Lee R, Muller I, Ezra D, Dayan M, et al. New Insights into the pathogenesis and non-surgical management of Graves' orbitopathy. Nat Rev Endocrinol. Accepted for publication.

24. Kozdon K, Fitchett C, Rose GE, Ezra DG, Bailly M. Mesenchymal stem cell-like properties of orbital fibroblasts in Graves' orbitopathy. Investig Ophthalmol Vis Sci. 2015;56:5743-50. https://doi.org/10.1167/iovs.15-16580.

25. Brandau S, Bruderek K, Hestermann K, Görtz GE, Horstmann M, Mattheis S, et al. Orbital fibroblasts from Graves' orbitopathy patients share functional and immunophenotypic properties with mesenchymal stem/stromal. Cells Investig Ophthalmol Vis Sci. 2015;56:6549-57. https://doi.org/10.1167/iovs.15-16610.

26. Smith TJ. Insights into the role of fibroblasts in human autoimmune diseases. Clin Exp Immunol. 2005;141:388-97. https:// doi.org/10.1111/j.1365-2249.2005.02824.x.

27. Alonso-Merino E, Martín-Orozco R, Ruíz-Llorente L, MartínezIglesias O, Velasco-Martín J, Montero-Pedrazuela A, et al. Thyroid hormones inhibit TGF- $\beta$ signaling and attenuate fibrotic responses. PNAS. 2016;113:E3451-E3460. https://doi.org/10. 1073/pnas.1506113113. 
28. Zhang L, Baker G, Janus D, Paddon C, Fuhrer D, Ludgate M. Biological effects of thyrotropin receptor activation on human orbital preadipocytes. Investig Ophthalmol Vis Sci. 2006;47:5197-203. https://doi.org/10.1167/iovs.06-0596.

29. Kumar S, Nadeem S, Stan MN, Coenen M, Bahn RS. A stimulatory TSH receptor antibody enhances adipogenesis via phosphoinositide 3-kinase activation in orbital preadipocytes from patients with Graves' ophthalmopathy. J Mol Endocrinol. 2011;46:155-63. https://doi.org/10.1530/jme-11-0006.

30. Zhang L, Bowen T, Grennan-Jones F, Paddon C, Giles P, Webber J, et al. Thyrotropin receptor activation increases hyaluronan production in preadipocyte-fibroblasts; contributory role in hyaluronan accumulation in thyroid dysfunction. J Biol Chem. 2009;284:26447-55.

31. Hagood JS. Thy-1 as an integrator of diverse extracellular signals. Front Cell Dev Biol. 2019. https://doi.org/10.3389/fcell.2019. 00026.

32. Koumas L, Smith TJ, Feldon S, Blumberg N, Phipps RP. Thy-1 expression in human fibroblast subsets defines myofibroblastic or lipofibroblastic phenotypes. Am J Pathol. 2003 ;163:1291-300.

33. Hu P, Barker TH. Thy-1 in integrin mediated mechanotransduction front. Cell Dev Biol. 2019. https://doi.org/10.3389/fcell.2019. 00022 .

34. Li H, Fitchett C, Kozdon K, Jayaram H, Rose GE, Bailly M, et al. Independent adipogenic and contractile properties of fibroblasts in Graves' orbitopathy: an in vitro model for the evaluation of treatments. PLoS ONE. 2014;9:e95586. https://doi.org/10.1371/ journal.pone.0095586.

35. Yang IH, Rose GE, Ezra DG, Bailly M. Macrophages promote a profibrotic phenotype in orbital fibroblasts through increased hyaluronic acid production and cell contractility. Sci Rep. 2019;9:9622 https://doi.org/10.1038/s41598-019-46075-1.

36. Shin MS, Lee N, Kang I. Effector T cell subsets in systemic lupus erythematosus: update focusing on Th17 cells. Curr Opin Rheumatol. 2011;23:444-8. https://doi.org/10.1097/BOR.0b013e3283 $49 \mathrm{a} 255$.

37. Fang S, Huang Y, Wang S, Zhang Y, Luo X, Liu L, et al. IL-17A exacerbates fibrosis by promoting the proinflammatory and profibrotic function of orbital fibroblasts in TAO. J Clin Endocrinol Metab. 2016;101:jc.2016-1882.
38. Fang S, Huang Y, Zhong S, Li Y, Zhang Y, Li Y, et al. Regulation of orbital fibrosis and adipogenesis by pathogenic Th17 cells in Graves orbitopathy. J Clin Endocrinol Metab. 2017;102:4273-83. https://doi.org/10.1210/jc.2017-01349.

39. Lee GR. The balance of Th17 versus treg cells in autoimmunity. Int J Mol Sci. 2018;19:730. https://doi.org/10.3390/ijms19030730.

40. Wucherpfennig KW. Mechanisms for the induction of autoimmunity by infectious agents. J Clin Investig. 2001;108: 1097-104. https://doi.org/10.1172/JCI14235.

41. Köhling H, Plummer SF, Marchesi JR, Davidge KS, Ludgate M. The microbiota and autoimmunity: their role in thyroid autoimmune diseases. Clin Immunol. 2017;183:63-74. https://doi.org/ 10.1016/j.clim.2017.07.001.

42. Covelli D, Ludgate M. The thyroid, the eyes and the gut: a possible connection. J Endocrinol Investig. 2017. https://doi.org/10. 1007/s40618-016-0594-6.

43. Many M-C, Costagliola S, Detrait M, Denef J-F, Vassart G, Ludgate M. Development of an animal model of autoimmune thyroid eye disease. J Immunol. 1999;162:4966-74.

44. Baker G, Mazziotti G, von Ruhland C, Ludgate M. Re-evaluating thyrotropin receptor induced mouse models of Graves' disease \& ophthalmopathy. Endocrinology. 2005;146:835-44.

45. Masetti G, Moshkelgosha S, Köhling H-L, Covelli D, Banga JP, Berchner-Pfannschmidt $\mathrm{U}$, et al. Gut microbiota in experimental murine model of Graves' orbitopathy established in different environments may modulate clinical presentation of disease. Microbiome. 2018;6:97. https://doi.org/10.1186/s40168-0180478-4; https://microbiomejournal.biomedcentral.com/track/pdf/ 10.1186/s40168-018-0478-4.

46. Ohtani N, Kawada N. Role of the gut-liver axis in liver inflammation, fibrosis, and cancer: a special focus on the gut microbiota relationship. Hepatol Commun. 2019;3:456-70. https://doi.org/10. 1002/hep4.1331.

47. Savin Z, Kivity S, Yonath H, Yehuda S. Smoking and the intestinal microbiome. Arch Microbiol. 2018;200:677-84.

48. Liu X, Wong SS, Taype CA, Kim J, Shentu TP, Espinoza CR, et al. Thy-1 interaction with Fas in lipid rafts regulates fibroblast apoptosis and lung injury resolution. Lab Investig. 2017;97: 256-67. 